\title{
ShapeCoder: A New Method for Visual Quantification of Body Mass Index in Young Children
}

Byoung-Keon Daniel Park, $\mathrm{PhD}^{1}$, Matthew P. Reed, $\mathrm{PhD}^{1,2}$, Niko Kaciroti, $\mathrm{PhD}^{3}$, Margaret Love, $\mathrm{BA}^{3}$, Alison L. Miller, $\mathrm{PhD}^{3,4}$, Danielle P. Appugliese, $\mathrm{MPH}^{5}$, Julie C. Lumeng, MD ${ }^{3,6,7}$

\section{Affiliations:}

${ }^{1}$ University of Michigan Transportation Research Institute, University of Michigan, Ann Arbor, Michigan; ${ }^{2}$ Department of Industrial and Operations Engineering, University of Michigan, Ann Arbor, Michigan; ${ }^{3}$ Center for Human Growth and Development, University of Michigan, Ann Arbor, Michigan; ${ }^{4}$ Department of Health Behavior and Health Education, School of Public Health, University of Michigan, Ann Arbor, Michigan; ${ }^{5}$ Appugliese Professional Advisors, LLC, North Easton, Massachusetts; ${ }^{6}$ Human Nutrition Program, Department of Environmental Health Sciences, School of Public Health, University of Michigan, Ann Arbor, Michigan;

${ }^{7}$ Department of Pediatrics, University of Michigan, Ann Arbor, Michigan

Address correspondence to: Julie Lumeng, MD; 300 North Ingalls Street, $10^{\text {th }}$ Floor, University of Michigan, Ann Arbor, Michigan, 48109-5406; Tele: 734-647-1102; Fax: 734936-9288; jlumeng@umich.edu

Running title: A New Method for Visual Quantification of BMI

Key Words: anthropometry, body image, obesity

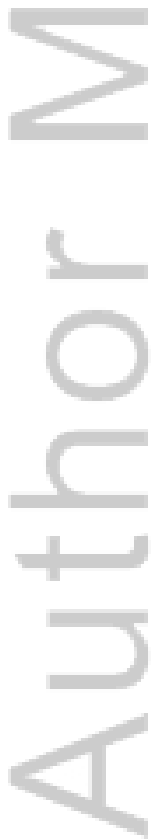

This is the author manuscript accepted for publication and has undergone full peer review but has not been through the copyediting, typesetting, pagination and proofreading process, which may lead to differences between this version and the Version of Record. Please cite this article as doi: 10.1111/ijpo.12202

This article is protected by copyright. All rights reserved. 
What is already known about this subject:

- Correctly estimating children's body mass index (BMI) visually is very difficult.

- Current approaches to visual rating have employed two-dimensional silhouettes.

- Ratings using two-dimensional silhouettes have modest accuracy against measured BMI.

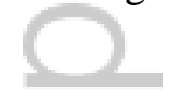

What this study adds:

- A rating system based on three-dimensional body shapes provides a method for estimating children's BMI that has high inter-rater reliability, fair sensitivity and good specificity for overweight/obesity and obesity.

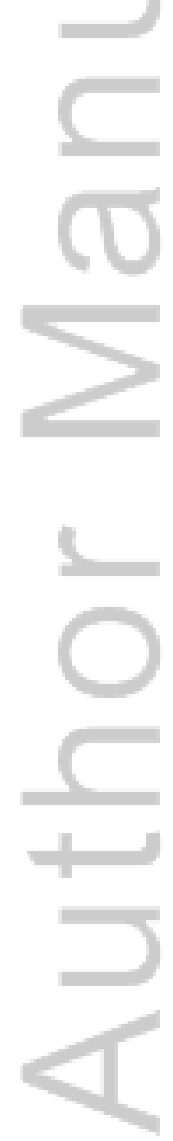

This article is protected by copyright. All rights reserved. 


\begin{abstract}
Background/Objective: To examine the inter-rater reliability and validity (sensitivity and specificity for overweight/obesity and obesity) of a three-dimensional visual rating system to quantify body mass index (BMI) in young children.

Subjects/Methods: Children $(n=242$, mean age 5.9 years, $50.0 \%$ male; $40.5 \%$ overweight/ obese) participated in a videotaped protocol with their parent and weight and height were measured. Research staff applied a novel three-dimensional computer-based figure rating system (ShapeCoder) to the child's videotaped image. Inter-rater reliability was calculated, as well as correlation with measured BMI and sensitivity, specificity, positive predictive value, and negative predictive value for overweight/obesity and obesity categorized based on the US Centers for Disease Control growth charts using measured BMI.

Results: Inter-rater reliability was excellent (intraclass correlation coefficient $=.98$ ). The correlation of ShapeCoder-generated BMI with measured BMI was .89. For overweight/obesity, the sensitivity, specificity, positive predictive value, and negative predictive value were $62 \%$, $97 \%, 94 \%$, and $79 \%$, respectively. For obesity, these values were $65 \%, 99 \%, 97 \%$, and $92 \%$, respectively.
\end{abstract}

Conclusion: ShapeCoder provides a method to quantify child BMI from video images with high inter-rater reliability, fair sensitivity, and good specificity for overweight/obesity and obesity. The approach offers an improvement over existing two-dimensional rating scales for BMI. 


\section{INTRODUCTION}

Estimating the body mass index (BMI) of adults using a rating scale of two-dimensional (2-D) silhouette drawings is possible with reasonable validity (e.g., $r=.70$ to .80$).{ }^{1-3}$ However, estimating the BMI of children using 2-D silhouette drawings has proven to be more challenging. A child's selection of one of seven 2-D photographic images of children of varying BMIs correlated with the image that their measured BMI would match only modestly $(r=.43$ in one study $^{4}$ and .56 among girls and .29 among boys in another study ${ }^{5}$ ). In one study, only $40.3 \%$ of mothers were able to correctly match their child's measured BMI to one of seven 2-D silhouette drawings. ${ }^{6}$ In another study with a separate set of seven 2-D drawings, sensitivity of the drawings for overweight/obesity based on parental selection was $70 \%$ and specificity was $79 \%{ }^{7}$

To address the limited validity of 2-D silhouette rating scales as an estimation of children's BMI, ShapeCoder, a new software tool based on three-dimensional (3-D) laser scans of child body shape was developed. ShapeCoder guides a rater through a series of rotatable, 3-D figures of standing children generated from a Statistical Body Shape Model (SBSM). ${ }^{8}$ At each of four levels, the rater chooses the ShapeCoder figure that best matches the body shape of the subject. The underlying SBSM associates each presented figure with a BMI.

This study sought to determine the reliability and validity of ShapeCoder for predicting child BMI from videotaped images of children. We hypothesized that ShapeCoder could be applied with acceptable inter-rater reliability, that it would be capable of generating BMI values that closely correlate with actual measured BMIs, and that it would be highly sensitive and 
specific for child overweight/obesity and obesity by measured BMI. Although BMI has wellknown limitations as an index of body fat, ${ }^{9}$ it is easy to measure and calculate, and is commonly used to define overweight/obesity and obesity. We therefore used BMI as the index of child adiposity against which to validate ShapeCoder.

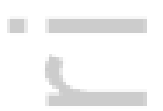

\section{METHODS}

\section{Participants}

A non-probability convenience sampling method was used to recruit participants. The study population included 242 children recruited from Head Start programs (free, federallysubsidized preschool programs for children from families earning a low income) in the Midwestern region of the United States who were part of a longitudinal study examining stress, eating behavior, and children's growth. Those meeting the following criteria were eligible for inclusion: caregiver had less than a four-year college degree, caregiver fluent in English, child born at 35 weeks gestation or more and without significant perinatal or neonatal complications, child without serious medical problems, and child not in foster care. To be included in this analysis, children also needed to have a measured BMI, which resulted in the exclusion of one child who declined anthropometry. In addition, one child whose measured BMI was a significant outlier at 38.46 and was excluded. The work described here represents a secondary analysis of videotaped parent-child interactions around feeding which were part of a follow-up study of this cohort occurring two years later that included an extensive data collection protocol 
focused on maternal feeding beliefs and practices. For the overall protocol, families attended two two-hour study visits in a private room at a local community center and provided three videotapes of mealtimes in their home. During study visits, families completed interviews, questionnaires, and the videotaped protocol described below. Participants were each compensated \$150. All parents gave written informed consent, children provided assent, and the University of Michigan Institutional Review Board approved the study. The sample of children was 50.0\% male and 43.8\% Hispanic or not white, and mean child age was 5.91 years (SD 0.71, range 4.0 to 8.1$)$. The majority $(90 \%)$ were from families earning a low income defined as having an income-to-needs ratio less than $185 \%$ of the federal poverty line.

\section{Videotaping Protocol}

Children were videotaped seated at a table with their parent while participating in a protocol designed to examine eating and feeding behaviors, which has been previously described. ${ }^{10}$ Briefly, each parent-child dyad was presented with a series of servings of food, invited to taste them if they wished, and then left alone in the room with the food for four minutes, during which the parent-child interaction was videotaped. The researcher returned to the room after each four minute period and asked the parent and child a series of standardized questions about their impressions of the food. In total, each videotape lasted about 20 minutes. The vast majority of children sat next to the parent throughout the task, generally engaging in a conversation with the parent about the food, and often moving around frequently in their chairs. Some children occasionally stood up next to their chairs. The camera was set up to capture the 
child's entire body on video, while also being as close as possible in order to allow assessment of facial expressions for other elements of the study.

\section{Anthropometry}

Research staff participated in a half-day training in research-grade anthropometry, were certified for appropriate technique, participated in quarterly refresher sessions regarding appropriate technique, and were recertified annually. A Detecto Portable Scale Model \# DR550 was used to weigh the children and a Seca 217 portable stadiometer was used to measure the children's height. A standard weight was used to check the calibration of the scale monthly. Children were measured according to the following procedures: Shoes and heavy clothing were removed. Children were weighed twice, and if the readings differed by more than $0.1 \mathrm{~kg}$, they was weighed two additional times. Children's heights were measured twice after verifying appropriate position and posture, and if the measurements were inconsistent by more than 0.5 $\mathrm{cm}$, they were measured two more times. The average of the final two measures was used for analysis. BMI was calculated as weight in kilograms divided by height in meters squared $(\mathrm{BMI}=$ weight in kilograms/(height in meters $)^{2}$ ), and BMI z-scores were derived from the age- and sexspecific US Centers for Disease Control growth charts. ${ }^{11}$ We elected to use these growth charts given that they provide a reference for US children, consistent with the nationality of children enrolled in this study. Child overweight/obese was defined as BMI $\geq 85^{\text {th }}$ percentile and obese was defined as $\mathrm{BMI} \geq 95^{\text {th }}$ percentile for age and sex.

\section{ShapeCoder Software}

This article is protected by copyright. All rights reserved. 
ShapeCoder software was developed by the authors based on data drawn from a separate sample of 73 boys and 67 girls ages three to 11 years with height range of 100 to $166 \mathrm{~cm}$ and BMI from 12 to $27 \mathrm{~kg} / \mathrm{m}^{2}$. High-resolution body surface data were obtained in a standing posture using a laser scanner. A standardized template was fit to the data and a principal component analysis was conducted to obtain a reduced-dimension representation. ${ }^{12}$ Linear regression analysis was conducted to predict body size and shape using standard anthropometric values as predictors. The age range of the subject pool (3 to 11 years old) was determined based on developmental maturity sufficient to stand still for 15 seconds at a time. The selected children were free of evident skeletal deformities, musculoskeletal injuries, or disabilities.

The ShapeCoder software generates 3-D figures using a SBSM ${ }^{17}$ based on these data (Figure 1). At each of four levels, five figures are shown and the rater chooses the one most similar to the study participant. At the first level, the figures are generated based on evenly spaced heights from $100 \mathrm{~cm}$ to $160 \mathrm{~cm}$. The figures are always shown with the same vertical dimension on the screen, so height is visible as body proportions rather than vertical extent on the screen (i.e., how much of the computer screen is occupied by the figure). The second level uses the height selected at the first level and presents five figures with BMIs ranging from 10 to $30 \mathrm{~kg} / \mathrm{m}^{2}$. The third level presents a narrower range of height $( \pm 10 \mathrm{~cm})$ based on the initial selection in Level 1, with the BMI held at the level selected in Level 2. In Level 4, the refined height selected in Level 3 is fixed, and BMI is varied $\pm 3 \mathrm{~kg} / \mathrm{m}^{2}$ relative to the value selected in Level 2. Hence, the best possible precision of ShapeCoder is $0.75 \mathrm{~kg} / \mathrm{m}^{2}$, or half of the final BMI 
interval. ShapeCoder exports the height and BMI associated with the figure selected in Level 4 for subsequent analysis. Supplementary information (a demonstration of ShapeCoder) is available at http://shapecoder.org.

\section{Application of ShapeCoder}

Two research staff members applied ShapeCoder to the videotaped images. Coders reviewed the ShapeCoder program manual and used it for a practice set of five videos independently, and then together, to gain experience using the program. A total of 15 videos (6\% of all videos) were coded by these two raters, from which inter-rater reliability on Shapecoder was calculated. It took three to four minutes to code each participant's body shape from the videotaped image.

\section{Statistical Analyses}

Data were analyzed with SAS 9.3 (SAS Institute, Cary, NC). To test the hypothesis that ShapeCoder could be applied with acceptable inter-rater reliability we calculated intraclass correlation coefficients (ICC) for the ShapeCoder-generated BMIs resulting from each of the two coders' application of the program.

Validity of ShapeCoder was assessed by examining the correlation of ShapeCodergenerated BMI with BMI by anthropometry, as well as the sensitivity and specificity for overweight/obesity and obesity. To test the hypothesis that ShapeCoder would generate a BMI value that closely correlated with actual measured BMI, we calculated Pearson's correlation coefficient for ShapeCoder-generated BMI against measured BMI. To test the hypothesis that 
ShapeCoder-generated BMIs would be highly sensitive and specific for overweight/obesity and obesity by measured BMI, we first transformed both ShapeCoder-generated BMIs and actual measured BMIs to BMI percentiles based on the US Centers for Disease Control growth charts. ${ }^{11}$ We then used these BMI percentiles to categorize children as overweight/obese or obese based on ShapeCoder as well as based on actual anthropometry. We then calculated the sensitivity, specificity, positive predictive value, and negative predictive value for ShapeCoder-generated BMI as a test for overweight/obesity and obesity as determined by BMI percentile based on US Centers for Disease Control growth charts. ${ }^{11}$

\section{RESULTS}

The mean measured BMI was 17.22 (SD 2.67) (range 12.68 to 32.01 ). The mean measured BMI z-score, based on the US Centers for Disease Control growth charts, ${ }^{11}$ was 0.80 (SD 1.01) (range -2.58 to 3.08). A total of $59.5 \%$ of children were not overweight (BMI $<85^{\text {th }}$ percentile), $20.25 \%$ were overweight $\left(85^{\text {th }}\right.$ percentile $\leq$ BMI $<95^{\text {th }}$ percentile), and $20.25 \%$ were obese (BMI $\geq 95^{\text {th }}$ percentile). The mean ShapeCoder-generated BMI was 16.50 (SD $2.51)($ range 13.00 to 26.50$)$.

There was support for excellent inter-rater reliability on the 15 videos coded by both raters: $\mathrm{ICC}=0.98$. With regard to validity of ShapeCoder, ShapeCoder-generated BMI showed a very strong correlation with measured BMI $(\mathrm{r}=.89, \mathrm{p}<.001)$. Measured BMI was predicted by Shapecoder BMI by the equation: Measured BMI $=1.65+0.94 *$ Shapecoder BMI (Figure 2). The R-square for this regression is .79 and the root mean squared error is $1.24 \mathrm{~kg} / \mathrm{m}^{2}$. 
The sensitivity and specificity of ShapeCoder-generated BMI for overweight/obesity by measured BMI were $62 \%$ and $97 \%$, respectively. The positive predictive value of ShapeCodergenerated BMI for overweight/obesity was $94 \%$. The negative predictive value of ShapeCodergenerated BMI for overweight/obesity was $79 \%$.

The sensitivity and specificity of ShapeCoder-generated BMI for obesity by measured BMI were $65 \%$ and $99 \%$, respectively. The positive predictive value of ShapeCoder-generated BMI for obesity was $97 \%$. The negative predictive value of ShapeCoder-generated BMI for obesity was $92 \%$.

Analyses stratified by child sex did not demonstrate meaningful differences.

\section{DISCUSSION}

This study provides evidence that a new approach for assessing children's BMI using a visual rating can be applied with high inter-rater reliability. The correlation of ShapeCodergenerated BMI with measured BMI $(r=.89)$ exceeded correlations reported in prior studies (albeit with child raters), which were all less than $.60 .{ }^{4,5}$ In the single prior study in which sensitivity and specificity for overweight/obesity were reported, ShapeCoder demonstrated somewhat weaker sensitivity $\left(62 \%\right.$ versus $\left.70 \%^{7}\right)$ but superior specificity $\left(97 \%\right.$ vs. $\left.79 \%^{7}\right)$.

ShapeCoder provides fair sensitivity for overweight/obesity and obesity, and good specificity. Thus, although ShapeCoder will only identify about $62 \%$ of children who are overweight/obese and $65 \%$ of those who are obese, it correctly identifies children as not overweight/obese $97 \%$ of the time, and correctly identifies children as not obese $99 \%$ of the 
time. When a child is identified as overweight/obese by ShapeCoder, $94 \%$ of the time the child is overweight/obese by anthropometry. When a child is identified as not overweight/obese by ShapeCoder, $79 \%$ of the time the child is indeed not overweight/obese. When the child is identified as obese by ShapeCoder, $97 \%$ of the time the child is obese. When the child is identified as not obese by ShapeCoder, $92 \%$ of the time the child is not obese.

ShapeCoder could be a valuable addition to extant tools that evaluate body type, particularly in large, epidemiologic studies of children that often do not include measured weight and height but sometimes do include videotaped images. ShapeCoder could also be used in research studies examining body image and eating disorders. As in any study, the choice of instrument will depend on whether the costs associated with false positives versus false negatives are higher or lower in a particular usage context.

There are several limitations to our study. The sample of children used to generate the SBSM in ShapeCoder was relatively small, primarily white, and with a smaller prevalence of overweight and obesity than among the children who were the participants in the current analysis. The SBSM also combines data from boys and girls. The age range of children in the coded sample of videotapes was constricted to 4.0 to 8.1 years, and it is not known if the results apply to children outside of this age range. There were no meaningful differences in our results by child sex, and differences could emerge within a sample of older children. The validity of ShapeCoder among children who have entered puberty is unknown. It is also unknown if the results apply to children of differing sociodemographic characteristics. This study also used 3-D 
moving images of fully clothed, seated children on videotape using a stationary camera; reliability and validity of ShapeCoder may have been higher if the children were viewable standing, from all angles, and with less clothing. In addition, although BMI is used in clinical practice to estimate adiposity, it has substantial limitations in accurately identifying overweight/obesity and obesity. Finally, the selection of levels in the software and the separation between adjacent images may have influenced the accuracy and precision of the results. For example, using a narrower range of BMIs in the final level may have improved the results. However, pilot testing suggested that it was not generally feasible to distinguish between figure images separated by less than $1.5 \mathrm{~kg} / \mathrm{m}^{2}$ (see Figure 1).

There are a number of directions for future work. First, confirmatory work in larger populations with varying degrees of adiposity (including larger groups of thinner or underweight children) and diverse race/ethnicity would be beneficial. In addition, sex-specific models for older children would be useful. Future work should also use methods for measuring adiposity that have greater precision than BMI, so that the validation of ShapeCoder-generated categorizations of overweight/obesity and obesity could be carried out against a measure that is a more accurate index of adiposity than BMI. Prior work has described parents' inability to accurately identify their child's own body type, ${ }^{6,7}$ but this work has been limited to the use of 2D figure ratings. If parents can accurately apply ShapeCoder to assess their own child's body shape, this would suggest that the prior literature concluding that parents cannot accurately recognize their child's body type may be an artifact of the limitations of 2-D rating scales. In 
summary, ShapeCoder can be used by researchers as a measure of children's BMI or weight status.

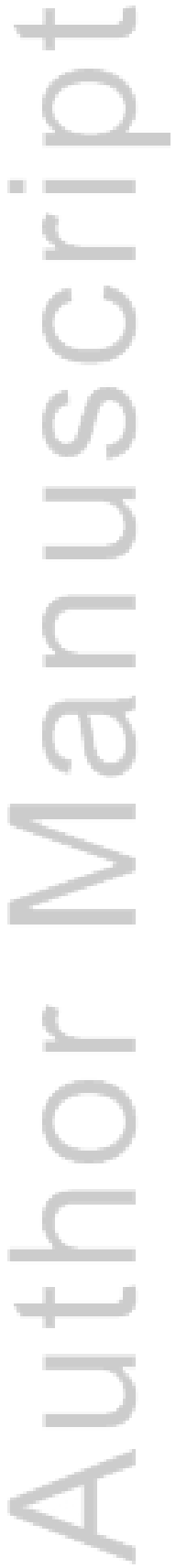

This article is protected by copyright. All rights reserved. 


\section{CONFLICT OF INTEREST}

The authors have no financial relationships relevant to this article to disclose. The authors have no conflicts of interest to disclose.

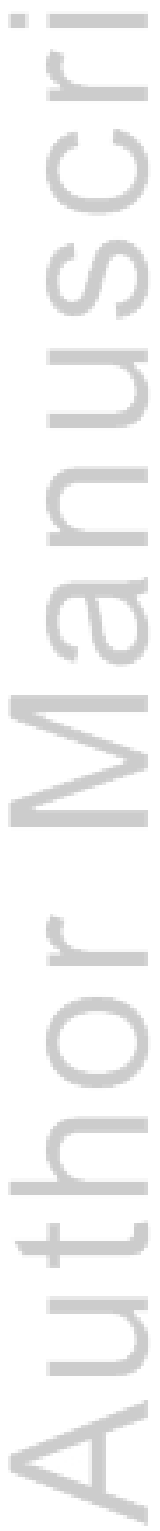

This article is protected by copyright. All rights reserved. 


\section{ACKNOWLEDGMENTS}

BP created the software, contributed to data analysis and interpretation, and helped to write the manuscript. MR created the software, contributed to data analysis and interpretation, and helped to write the manuscript. NK contributed to data analysis and interpretation and helped to write the manuscript. ML contributed to coding of body shape, conducted the literature search, and helped to write the manuscript. AM contributed to data collection, analysis and interpretation, and helped to write the manuscript. DA contributed to data analysis and helped to write the manuscript. JL conceived of the study design, contributed to the data collection and interpretation, helped to conduct the literature search, and helped to write the manuscript.

The work was supported by NIH 5R01HD06135 and the MCubed program at the University of Michigan.

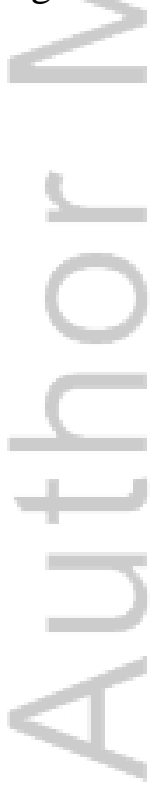

This article is protected by copyright. All rights reserved. 


\section{REFERENCES}

1. Bulik C, Wade T, Heath A, Martin N, Stunkard A, Eaves L. Relating body mass index to figural stimuli: Population-based normative data for Caucasians. Int J Obes 2001; 25: 1517-24.

2. Sorensen T, Stunkard A, Teasdale T, Higgins M. The accuracy of reports of weight: Children's recall of their parents' weights 15 years earlier. Int J Obes 1983; 7: 115-22.

3. $=$ Cardinal TM, Kaciroti N, Lumeng JC. The figure rating scale as an index of weight status of women on videotape. Obesity 2006; 14(12): 2132-5.

4. Saxton J, Hill C, Chadwick P, Wardle J. Weight status and perceived body size in children. Archives of Disease in Childhood 2009; 94(12): 944-949.

5. Truby H, Paxton SJ. Development of the Children's Body Image Scale. Br J Clin Psychol 2002; 41(Pt 2): 185-203.

6. Warschburger P, Kröller K. Maternal Perception of Weight Status and Health Risks Associated With Obesity in Children. Pediatrics 2009; 124(1): e60-e68.

7. Eckstein KC, Mikhail LM, Ariza AJ, Thomson JS, Millard SC, Binns HJ. Parents' perceptions of their child's weight and health. Pediatrics 2006; 117(3): 681-90.

8. Park BK, Lumeng JC, Lumeng CN, Ebert SM, Reed MP. Child body shape measurement using depth cameras and a statistical body shape model. Ergonomics 2015; 58(2): 301-9.

9. Power C, Lake JK, Cole TJ. Measurement and long-term health risks of child and adolescent fatness. Int J Obes Relat Metab Disord 1997; 21(7): 507-26.

10. Goulding AN, Rosenblum KL, Miller AL, Peterson KE, Chen YP, Kaciroti N et al. Associations between maternal depressive symptoms and child feeding practices in a cross-sectional study of low-income mothers and their young children. Int J Behav Nutr Phys Act 2014; 11(75): 1479-5868.

11. Kuczmarski RJ, Ogden CL, Grummer-Strawn LM, Flegal KM, Guo SS, Wei R et al. CDC growth charts: United States. Advance data 2000; (314): 1.

12. Modeling variability in torso shape for chair and seat design. Proceedings of the ASME Design Engineering Technical Conferences; ASME, New York, 2008.

This article is protected by copyright. All rights reserved. 


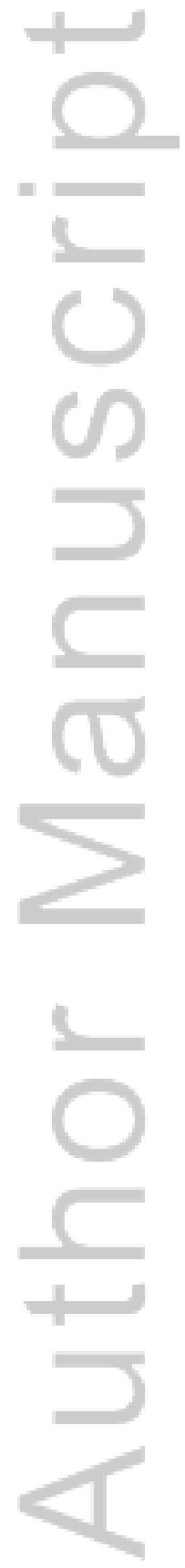

This article is protected by copyright. All rights reserved. 
Figure 1. Illustration of four sequential screens from ShapeCoder. At Level 1, the five figures represent body shapes based on evenly spaced heights from $100 \mathrm{~cm}$ to $160 \mathrm{~cm}$. The figure with the dot underneath it represents a hypothetical choice of the rater. At Level 2, the five figures represent a range of body types for the height selected at the first level (e.g., $115 \mathrm{~cm})$ with BMIs ranging from 10 to $30 \mathrm{~kg} / \mathrm{m}^{2}$. At Level 3, the five figures represent a narrower range of height ( \pm $10 \mathrm{~cm}$ ) based on the initial selection in Level 1, with the BMI held at the level selected in Level 2. At Level 4 , the five figures represent body shapes with BMI varied $\pm 3 \mathrm{~kg} / \mathrm{m}^{2}$ relative to the BMI value selected in Level 2 and the refined height selected in Level 3. The final highlighted choice has height of $110 \mathrm{~cm}$ and $26.5 \mathrm{~kg} / \mathrm{m}^{2}$. Note that each individual image can be rotated on the screen.

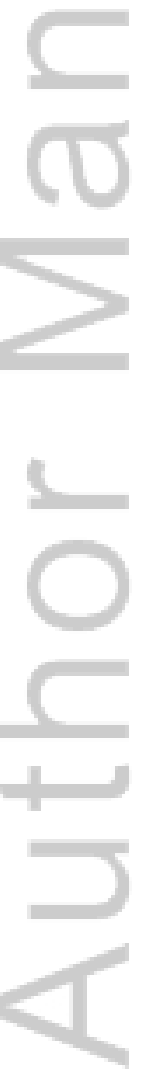

This article is protected by copyright. All rights reserved. 
Figure 2. Correlation of ShapeCoder-generated BMI with measured BMI. The regression equation is $\mathrm{y}=0.94 \mathrm{x}+1.65$.

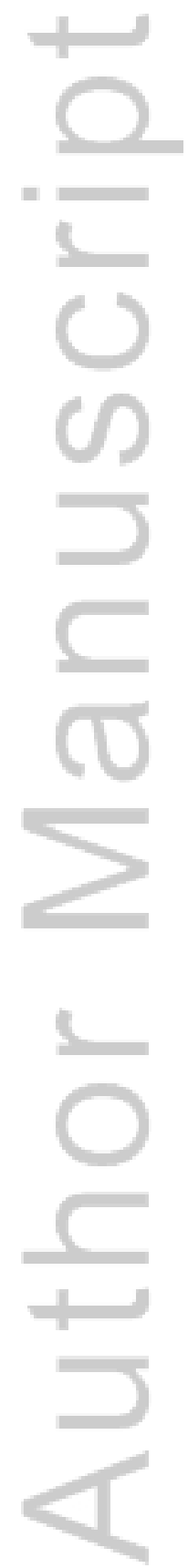

This article is protected by copyright. All rights reserved. 



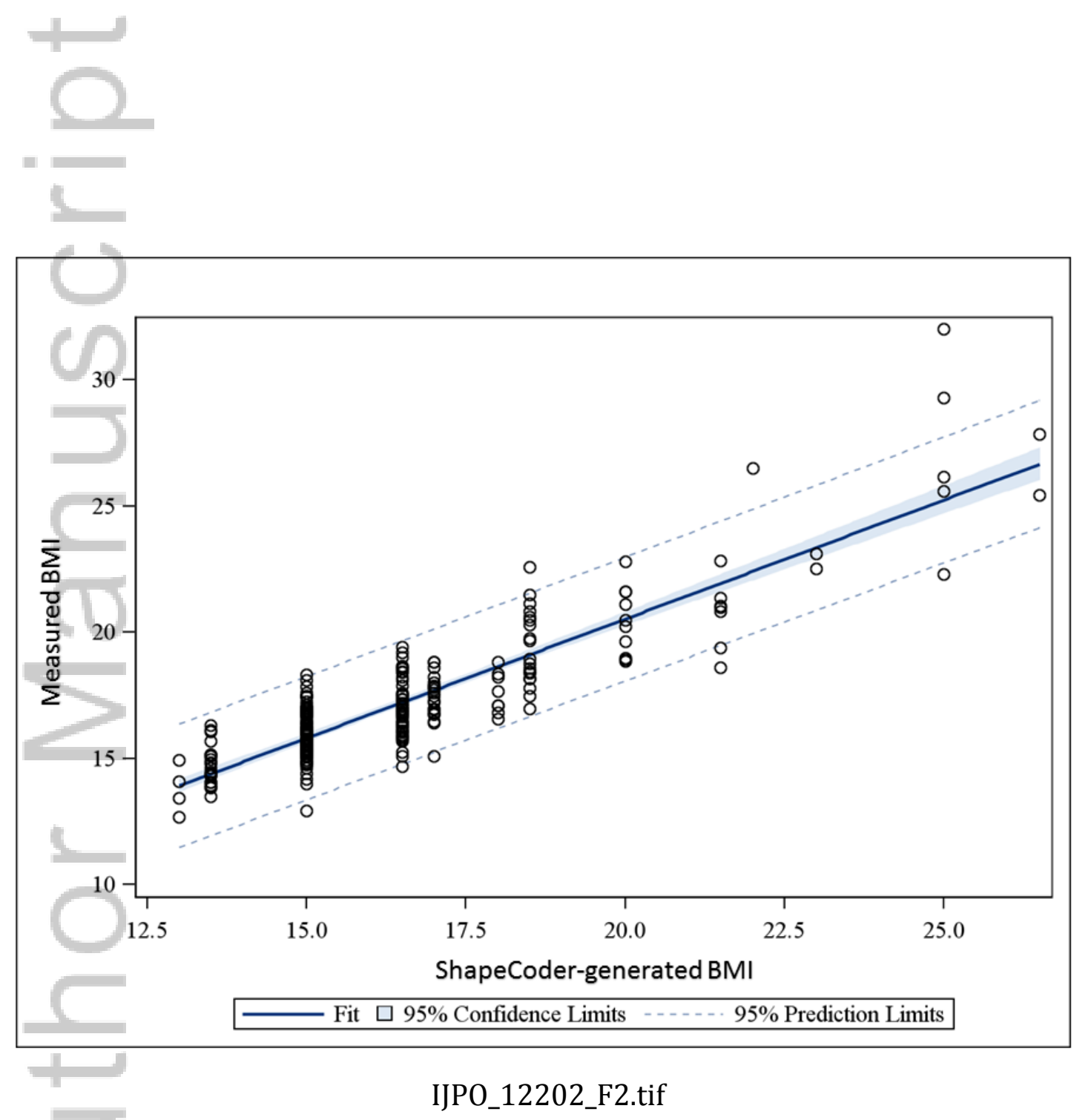

This article is protected by copyright. All rights reserved. 